\title{
Oral Pyogenic Granuloma: Case Report of an Atypical Presentation
}

\author{
Emory Byrd ${ }^{1}$, Jamie Bloss ${ }^{2}$, and Iquebal Hasan ${ }^{3 *}$ \\ ${ }^{1}$ D3 Student, ECU School of Dental Medicine, USA \\ ${ }^{2}$ Liaison Librarian, Allied Health Sciences and Dental Medicine, East Carolina University, USA \\ ${ }^{3}$ Clinical Assistant Professor, Oral Medicine, ECU School of Dental Medicine, USA
}

*Corresponding author: Iquebal Hasan, Clinical Assistant Professor, Oral Medicine, ECU School of Dental Medicine, USA.
Received Date: June 01, 2020

Published Date: June 10, 2020

\begin{abstract}
"Granulomas seem to be a defensive mechanism that triggers the body to "wall off" foreign invaders such as bacteria or fungi to keep them from spreading" [1]. Pyogenic granuloma is a relatively common benign skin growth that consists of vascular nodules of proliferating capillaries [2]. They grow rapidly and often occur following a minor injury or trauma, most likely as a vascular or fibrous response to injury or irritant [2]. Ultimately, the etiology of pyogenic granuloma is still unknown. Oral pyogenic granuloma is most commonly found on the gingiva, followed by the lips, tongue, buccal mucosa, and hard palate [3]. It often presents as a red, smooth, or lobulated exophytic lesion which can be pedunculated or sessile and often bleeds on provocation [4]. A microscopic evaluation of pyogenic granuloma often reveals that the lesion is fully or partially covered by parakeratotic or non-keratinized stratified squamous epithelium. The majority of the lesion is formed by a lobulated or non-lobulated mass of angiomatous tissue, with limited collagen present in the connective tissue of the lesion. The surface of the lesion may or may not be ulcerated but can be infiltrated by plasma cells, lymphocytes, and neutrophils [5]. Treatment of these lesions most often consists of surgical excision or curettage and electrodesication [2].
\end{abstract}

\section{Case Report}

This case report is about a 54-year-old Caucasian woman who presented to the ECU School of Dental Medicine (ECU Sodom) for treatment of a pedunculated palatal lesion on the left side of her hard palate oral candidiasis which was present throughout her mouth. The patient was referred to oral medicine specialist at ECU School of Dentistry for evaluation of a "skin tag" on the left side of the palate and white and red lesions which were in her entire mouth but mainly in the posterior hard palate. Her past medical history was significant for gastroesophageal reflux disorder, chronic obstructive pulmonary disease, anxiety, depression, diabetes, hypertension, myocardial infarction, stroke, and severe peripheral vascular disease. When she presented to the ECU SoDM Faculty Practice clinic, she was taking proventil, amlodipine, carvedilol, lisinopril, nitroglycerin, polyethylene glycol, vitamin D, klonopin, welbutrin, percocet, hydrochlorothiazide, plavix, aspirin, advair, diskus, gabapentin, albuterol, ferrous sulphate, Zanaflex, diazepam, cefdinir, and prednisone. The patient also reported that she smoked one pack of cigarettes a day for the past 38 years and uses recreational marijuana.

The patient was mainly concerned about the white areas throughout her mouth. She had previously been prescribed antibiotics and steroids for the white areas, but she believed they had made the white areas worse. She also reported that she had noticed that her voice had become hoarse sounding. Upon examination, the patient was edentulous and wore maxillary and mandibular dentures. She had severe dry mouth with lack of pooling of saliva in 
the anterior floor of her mouth. The white lesions were diagnosed as candidiasis and it was present in her entire mouth including the tongue and oropharynx. Her initial diagnosis was dry mouth and oral candidiasis secondary to dry mouth. The lesion on the left anterior palate was thin, smooth, and pedunculated. It measured $4 \mathrm{~mm}$ at the base, $6 \mathrm{~mm}$ at the widest area, and $8 \mathrm{~mm}$ in length. It was flat and wide and hung from its base, shaped similar to a paddle. It was attributed to frictional trauma from her denture.

The patient's treatment recommendations were over the counter dry mouth products, clotrimazole troches to treat the candidiasis. She was also told to contact her physician regarding stopping the antibiotic. She was also scheduled for biopsy of the "skin tag" after initial treatment with anti-fungal medication.

The patient returned for a follow-up appointment 21 days after her initial appointment. She reported significant improvement in oral dryness and in the white lesions. Examination revealed that there was improvement in lubrication in her mouth and the candidiasis had improved significantly as well but was still present. She was recommended to take an additional course of the antifungal medication and was scheduled for biopsy (Figure 1).

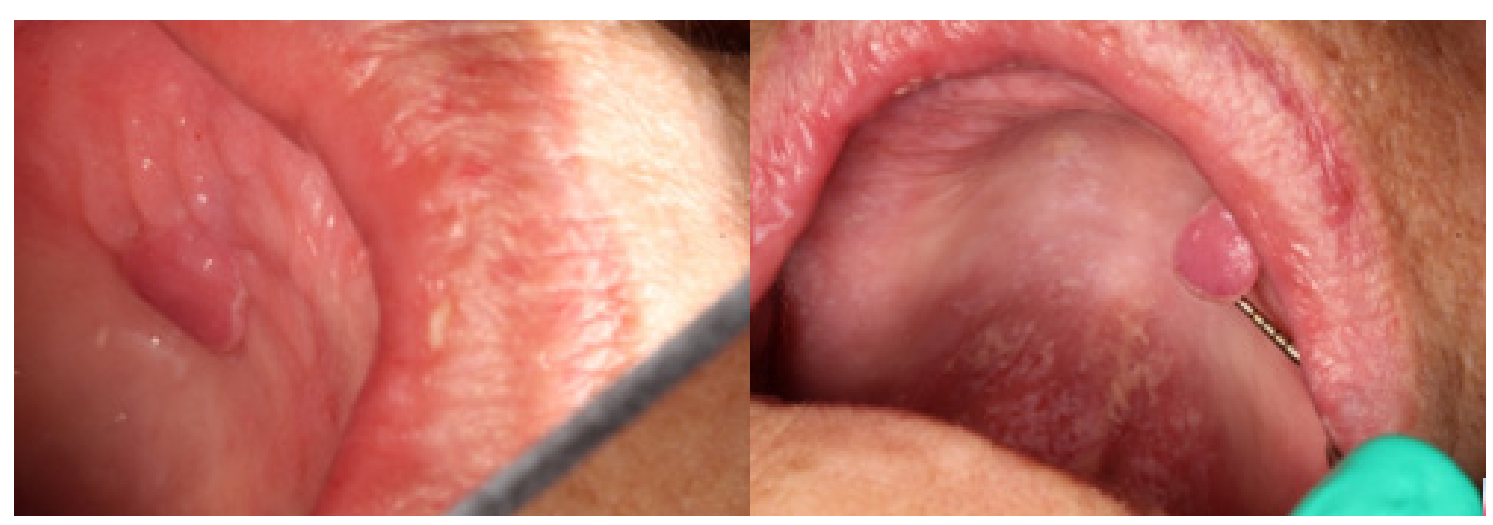

Figure 1

The patient presented for biopsy of the palatal lesion with the oral surgeon. Oral surgeons' clinical diagnosis was same as oral medicine specialist as traumatic fibroma. Local anesthesia of the area was achieved using one half of a carpule of lidocaine $2 \%$ 1:100,000 epinephrine. An excisional biopsy was performed using a 15-blade scalpel and eye loop cautery for hemostasis. The patient was recommended to return in two weeks for a post-surgical followup appointment and biopsy result with oral medicine specialist.

\section{Results}

The surgical pathology report obtained from the biopsy revealed that the lesion was pyogenic granuloma, and not a traumatic fibroma as it had initially been diagnosed. The microscopic examination displayed a focally ulcerated nodular fragment of oral mucosa, partially surfaced by hyperparakaratotic stratified squamous epithelium with a normal pattern of maturation, and a fibropurulent membrane. The underlying connective tissue was characterized by lobular aggregates of hyperplastic granulation tissue with numerous vascular channels contained within this stroma. An acute and chronic inflammatory cell infiltrate was observed throughout the histologic specimen, mainly composed of neutrophils, lymphocytes, plasma cells, and extravasated erythrocytes. There was no evidence of malignancy.

\section{Discussion}

Pyogenic granuloma is very common but what makes this case unique is its location and appearance. While oral pyogenic granuloma is relatively common, it develops most often on the gingiva. This also made the clinic diagnosis challenging even for two experienced clinicians with combined experience of over forty year. Histological examination was the only way it was possible. Since the patient wore a maxillary complete denture, it was initially assumed that the lesion was a fibroma caused by an ill-fitting denture. The lesion discussed in this case was smooth, fibrous and pedunculated in appearance. While these presentations are within the realm of normal appearance of typical pyogenic granuloma, this lesion was also flat and paddle-shaped, which is atypical of the exophytic nature of pyogenic granuloma. The lesion was also not erythematous and did not bleed upon provocation, a common occurrence in pyogenic granuloma. The uncharacteristic appearance of the lesion supported the initial fibroma diagnosis and not pyogenic granuloma.

The biopsy revealed an ulcerated lesion with hyperparakaratotic stratified squamous epithelium with normal maturation. The normal maturation of cells seen under microscopic evaluation indicated that the lesion was benign, ruling out malignant diagnoses. Similarly, the biopsy showed an abundance of vascular channels with inflammatory cell infiltrate including neutrophils, lymphocytes, plasma cells, and extravasated erythrocytes. This presentation is typical for microscopic appearance of pyogenic granuloma, thus confirming the diagnosis.

Recurrence can happen in pyogenic granuloma and the rate ranges from $0-15.8 \%$ of cases [6, 7]. Recurrence is more common 
in patients who develop pyogenic granuloma while pregnant and less common for patients who present with extragingival pyogenic granuloma [8]. The patient was informed of this possibility and told to monitor the area for recurrence of the lesion.

\section{Conclusion}

"Pyogenic granuloma" is a misnomer because the lesion does not contain pus and is not strictly speaking a granuloma [9]. Combination of poor oral hygiene and trauma causes one third of these lesions [9]. The visual presentation appeared similar to a traumatic fibroma, but the histological presentation distinguished the palatal lesion as pyogenic granuloma. This case emphasizes the importance of biopsy and histopathological examination in diagnosis of oral lesion. It also demonstrates the significance of managing oral dryness. Lack of lubrication in the mouth can contribute to poor oral hygiene and also cause trauma from denture. It may also contribute to oral fungal infection. In case of this patient the reduced salivary flow is most likely due to medications. This can be managed with over the counter dry mouth products. While taking care of the fungal infection and pyogenic granuloma was the primary reason for patients visit, it is also important in cases like to take care of the root cause of the problem.

\section{Acknowledgement}

None.

\section{Conflict of Interest}

No conflict of interest.

\section{References}

1. Granuloma: What does it mean?

2. Denise M Aaron (2019) Pyogenic Granulomas. Merck Manual.

3. Kamal R, Dahiya P, Puri A (2012) Oral pyogenic granuloma: Various concepts of etiopathogenesis. J Oral Maxillofac Pathol 16(1): 79-82.

4. Sachdeva SK (2015) Extra gingival Pyogenic Granuloma: An Unusual Clinical Presentation. J Dent (Shiraz) 16(3 Suppl): 282-285.

5. Pyogenic Granuloma. American Osteopathic College of Dermatology.

6. Bhaskar SN, Jacoway JR (1966) Pyogenic granuloma-clinical features, incidence, histology, and result of treatment: report of 242 cases. J Oral Surg 24(5): 391-398.

7. Lawoyin JO, Arotiba JT, Dosumu $O 0$ (1997) Oral pyogenic granuloma: A review of 38 cases from Ibadan, Nigeria. Br J Oral Maxillofac Surg 35(3): 185-189.

8. Sapp JP, Eversole LR, Wyoscki GP (1997) Contemporary oral and maxillofacial pathology ( $\left.2^{\text {nd }} e d n\right)$. USA, pp: 318-322.

9. Verma PK, Srivastava R, Baranwal HC, Chaturvedi TP, Gautam A, et al. (2012) Pyogenic Granuloma-Hyperplastic Lesion of Gingiva: Case Reports. Open Dental Journal 6: 153-156. 